Published in "Human Movement Science doi:10.1016/j.humov.2011.04.006, 2011" which should be cited to refer to this work.

\title{
Changes in predictive motor control in drop-jumps based on uncertainties in task execution
}

\author{
Christian Leukel ${ }^{\mathrm{a}, \mathrm{b}, *}$, Wolfgang Taube ${ }^{\mathrm{b}}$, Michael Lorch ${ }^{\mathrm{a}}$, Albert Gollhofer ${ }^{\mathrm{a}}$ \\ ${ }^{a}$ Department of Sport Science, University of Freiburg, Germany \\ ${ }^{\mathrm{b}}$ Department of Medicine, Unit of Sport Science, University of Fribourg, Switzerland
}

\begin{abstract}
Drop-jumps are controlled by predictive and reactive motor strategies which differ with respect to the utilization of sensory feedback. With reaction, sensory feedback is integrated while performing the task. With prediction, sensory information may be used prior to movement onset. Certainty about upcoming events is important for prediction. The present study aimed at investigating how uncertainties in the task execution affect predictive motor control in dropjumps. Ten healthy subjects ( $22 \pm 1$ years, $M \pm S D$ ) participated. The subjects performed either (i) drop-jumps by knowing that they might had to switch to a landing movement upon an auditory cue, which was sometimes elicited prior to touch-down (uncertainty). In (ii), subjects performed drop-jumps by knowing that there would be no auditory cue and consequently no switch of the movement (certainty). The m. soleus EMG prior to touch-down was higher when subjects knew there would be no auditory cue compared to when subjects performed the same task but switching from dropjump to landing was possible (uncertainty). The EMG was reversed in the late concentric phase, meaning that it was higher in the high uncertainty task. The results of the present study showed that the muscular activity was predictively adjusted according to uncertainties in task execution. It is argued that tendomuscular stiffness was the variable responsible for the adjustment of muscular activity. The required tendomuscular stiffness was higher in drop-jumps than in landings. Consequently, when it was not certain whether to jump or to land, muscular activity and therefore tendomuscular stiffness was reduced.
\end{abstract}

\footnotetext{
* Corresponding author at: Department of Sport Science, University of Freiburg, Schwarzwaldstr. 175, 79117 Freiburg Germany. Tel.: +49 761203 4515; fax: +497612034534.

E-mail address: christian.leukel@sport.uni-freiburg.de (C. Leukel).
} 


\section{Introduction}

Sensory information is important to execute motor tasks. Thereby, movement control can be categorized into two types which use sensory information differently. For the first type, muscular contraction(s) are planned and executed based on sensory information which may be integrated into the motor command prior to movement initiation (called predictive motor control). The second type of control is reactive because sensory feedback is used to contract the muscle while performing a motor task (Bastian, 2006). One of the simplest reactions are (spinal) reflexes (Sherrington, 1910). In most complex motor tasks, both of these types of movement control, often termed feedforward (predictive) and feedback (reactive), are interacting.

Drop-jumps and hoppings are a good example of the interaction of predictive and reactive motor control (Komi, 1984, 2003). These movements are characterized by (i) a pre-activation phase, (ii) an eccentric phase followed immediately by (iii) a concentric muscular action (Komi, 1984, 2003). The motor output in the pre-activation phase just prior to touch-down in drop-jumps and hoppings as well as part of the muscular activation after touch-down (in the eccentric phase) is predictive (Zuur et al., 2010). Part of the muscular activation after touch-down is reactive because spinal reflexes (e.g., the so called short latency response (SLR)) are elicited at touch-down and contribute to the motor output (Gollhofer \& Kyrolainen, 1991; Leukel et al., 2009; Zuur et al., 2010). This interaction of predictive and reactive motor control in drop-jumps and hoppings was hypothesized almost 40 years ago (Jones \& Watt, 1971) and demonstrated in a recent study where subjects hopped on a robotic platform which moved up or down while the subjects were airborne (Zuur et al., 2010). Part of the muscular activation after touch-down changed according to the time when the load was applied (the time when the subjects hit the ground and proprioceptive feedback was evoked). The activation prior to touch-down was time-locked independent of the instant of touch-down. The predictive (pre-activation) phase prior to touch-down is an important part in drop-jumps in order to adjust muscular stiffness (Komi, 2003). The muscular pre-activation is not only rigid with respect to timing but also with respect to amplitude in predictable (laboratory) settings (Zuur et al., 2010). However, in daily life, predictions have sometimes to be changed because of unexpected events forcing to adapt motor control. The aim of the present study was to investigate the adaptability of the predictive part of the muscular activation in dropjumps. Therefore, subjects were instructed to (i) perform drop-jumps or (ii) were told that they might have to switch to a landing movement based on an auditory cue presented in the flight phase prior to touch-down. The jump-off and the flight phase until touch-down were similar for drop-jumps and landings. The muscular activation, however, was shown to be higher in drop-jumps than in landings (Dyhre-Poulsen, Simonsen, \& Voigt, 1991; Hobara, Kanosue, \& Suzuki, 2007; Leukel, Gollhofer, \& Taube, 2008). Given the possibility that the task had to switch from drop-jumping to landing, it was interesting to see how the central nervous system adjusted the muscular activation.

\section{Methods}

A total of 10 subjects (aged $22 \pm 1$ years) without orthopaedic and neurological disorders participated. The study was approved by the local ethics committee of the Albert-Ludwigs-University in Freiburg and experimental procedures were performed in accordance with the Declaration of Helsinki. All subjects gave written informed consent prior to participation.

\subsection{EMG recordings}

EMG recordings were obtained from the leg extensor muscle soleus (SOL, right leg) which was shown to be differently activated in drop-jumps versus landings (Dyhre-Poulsen et al. 1991; Leukel et al., 2008). After preparation, bipolar surface electrodes (Blue sensor P, Ambu ${ }^{\circledR}$, Bad Nauheim, Germany) were attached to the skin $(2 \mathrm{~cm}$ inter-electrode distance). The reference electrode was placed on the tibial plateau. EMG signals were amplified $(\times 1000)$, bandpass-filtered $(10-1000 \mathrm{~Hz})$ and sampled at $4 \mathrm{kHz}$. The data were stored for offline analysis with custom built software (LabView ${ }^{\circledR}$ based, National Instruments ${ }^{\circledR}$, Austin, Texas). 
The ground reaction force $(\mathrm{Fz})$ was analyzed using a 3-dimensional force platform (AccuGait ${ }^{\circledR}$, AMTI, Watertown, USA) (Fig. 2).

\subsection{Landing/drop-jumping procedure}

The experiment started with a warm-up (hopping) for $5 \mathrm{~min}$. Thereafter, the subjects were instructed how to perform the drop-jumps and the landings during the experiment. Both movements (landings and drop-jumps) were executed from $50 \mathrm{~cm}$ falling height. The reason for using $50 \mathrm{~cm}$ was practically motivated: the time to jump from $50 \mathrm{~cm}$ was sufficiently long to present the auditory cue at $170 \mathrm{~ms}$ before reaching the ground (please see below: Section 2.3) but the height was sufficiently low enough to perform a reactive (drop-jump) movement. The optimal falling height to perform a drop-jump is, in most subjects, around $20-40 \mathrm{~cm}$. At around $90 \mathrm{~cm}$ falling height, subjects cannot perform a reactive movement anymore (Komi \& Gollhofer, 1997). Consequently, $50 \mathrm{~cm}$ falling height met the methodological demands of the present study without "losing" the reactive character of the drop-jump. Before drop-jumping, both arms should be held at the waist, the left leg remained stable in order to secure upright stance on the $50 \mathrm{~cm}$ platform, whereas the right leg was lifted and projected in front of the body. The jump-off was performed with the left leg. The purpose of this procedure was to keep the right leg (from which was recorded) relaxed until touch-down. Subjects were asked to touch-down on both feet and to jump-off the ground as quickly as possible. Additionally, subjects were instructed to keep the drop-jumping procedure similar throughout all drop-jumps with regard to the starting position on the platform, head position, and push off phase after landing. The position of each subject on the platform before drop-jumping was visually controlled by the experimenters. For landings, the procedure until touch-down was the same as that during drop-jumping. Subjects were told to rest for 2 min after every 20 drop-jumps/landings in order to avoid fatigue.

\subsection{Experimental protocol}

The subjects started either with landing ( 5 subjects) or drop-jumping ( 5 subjects). This order was reversed in the second half of the experiment, e.g., subjects who started with landings performed drop-jumps and vice versa. While landing/drop-jumping, an auditory cue ( $2 \mathrm{kHz}, 100 \mathrm{~ms})$ was sometimes presented at (a) $170 \mathrm{~ms}$, (b) $110 \mathrm{~ms}$, or (c) $50 \mathrm{~ms}$ before ground contact. The different conditions were randomized (one randomized cycle consisted of the 170/110/50 ms conditions, mixed with 3 conditions in which no cue was presented, making 6 in total). The auditory cues were triggered with custom built software (LabView ${ }^{\circledR}$ based, Austin, Texas, USA) using an optoelectric light barrier (Optojump ${ }^{\circledR}$, Microgate, Bolzano, Italy) as trigger signal. In order to set the delays after which the auditory cues were elicited with respect to the trigger signal from the light barrier, the individual time between passing the light barrier and ground contact was calculated. Ground contact was determined by analysing the ground reaction force. If there was an auditory cue while preparing to land/jump, the subjects were told to perform the opposite movement (from drop-jumping to landing and vice versa). When there was no auditory cue, the subjects performed the movement they were originally instructed to do. The reason for using these specific timings of the auditory cue was related to theoretical considerations about the capability of the central nervous system to process the sensory information, modify the prepared motor command, and finally activate the muscles of the lower leg. At $50 \mathrm{~ms}$ before ground contact, the time to successfully change the muscular pattern was expected to be too short. The conduction time of the fastest corticospinal fibers activated by the motor areas of the neocortex to contract the muscles of the lower leg is approximately $30 \mathrm{~ms}$ (e.g., Petersen, Christensen, Morita, Sinkjaer, \& Nielsen, 1998). This leaves the insufficient time of $20 \mathrm{~ms}$ to perceive the auditory cue, change the motor command and process this information to the motor output areas. When the auditory cue was given $60 \mathrm{~ms}$ earlier, at $110 \mathrm{~ms}$, we assumed that subjects are able to switch the task in a majority of the cases. At $170 \mathrm{~ms}$, again $60 \mathrm{~ms}$ earlier, the time was suggested to be sufficiently long to successfully switch the motor pattern.

Fifteen trials of each condition for both, drop-jumps and landings (where the primary instruction was to jump/land and to switch the movement when a cue was presented), were recorded. In addition to the trials with auditory cues, 15 control drop-jumps/landings were performed where the subjects 
knew that there would be no auditory cue and, consequently, that there would be no switch of the movement.

\subsection{Data analysis and statistics}

To analyze the muscular activity of the different phases of the landings/drop-jumps, root mean square (RMS) values of the unrectified SOL EMG were individually calculated for the following time windows: $-60-0 \mathrm{~ms}$ (whereas 0 refers to ground contact) for the pre-activation phase prior to ground contact, $0-60 \mathrm{~ms}$ for the eccentric phase after ground contact, $60-120 \mathrm{~ms}$, and $120-180 \mathrm{~ms}$, respectively, for the early and late concentric phase.

The drop-jump height was calculated using the formula: $h=1 / 8 \times g \times t^{2}$ in accordance with previous studies (Leukel, Taube, Gruber, Hodapp, \& Gollhofer, 2008; Leukel et al., 2008). Thereby, $g$ refers to acceleration of gravity and $t$ refers to the time of the flight phase.

Based on the design of the study, 4 conditions could be ascribed to both tasks-landings and dropjumps: in the first condition (termed "cue will not occur - CWNO") the subjects knew that there would be no auditory cue. Thus, they could perform the landings/drop-jumps without preparing to switch the motor pattern. The second, called "cue may occur - CMO", referred to the condition where the subjects performed the instructed movement (no switch in the motor pattern) but with the possibility that the movement could be switched. The third and fourth condition, termed " $170 \mathrm{~ms}$ " and "110 ms", respectively, referred to the landings/drop-jumps where the movement was successfully switched based on the auditory cue presented at 170 and $110 \mathrm{~ms}$ before ground contact.

In accordance with the aim of the present study, we specifically focused on the pre-activation phase of the drop-jumps in conditions where (i) subjects knew that there would be no switch of the movement (CWNO) and where (ii) subjects knew that they potentially have to switch the movement (CMO). Therefore, the EMG activity in the time period of $-60-0 \mathrm{~ms}$ was analyzed between CWNO and CMO by a paired Student's $t$-test. Furthermore, the EMG activity for all other time intervals (0-60 ms; 60-120 ms; $120-180 \mathrm{~ms}$ ) were analyzed with paired Student's $t$-tests. These $t$-tests were Bonferroni corrected.

To see whether there was a general difference in the muscular activation between all tested conditions (including conditions where subjects actually switched from landing to jumping and vice versa) the muscular activation during landings and drop-jumps was analyzed by means of a repeatedmeasures ANOVA with the within-subject factors time window (-60-0 ms; 0-60 ms; 60-120 ms; $120-180 \mathrm{~ms}$ ) and condition (CWNO, CMO, $170 \mathrm{~ms}, 110 \mathrm{~ms}$ ). The jump height and the ground contact time of the drop-jumps were analyzed using a repeated-measures ANOVA with the within-subject factor condition (CWNO, CMO, $170 \mathrm{~ms}, 110 \mathrm{~ms}$ ). The condition where the auditory cue was given $50 \mathrm{~ms}$ before touch-down ("50 ms") was not statistically analyzed because the subjects could not alter their motor pattern due to time constraints, i.e., the time was not sufficiently long to switch from one to the other movement. Differences were regarded significant at $p<.05$ for all tests. SPSS software 15.0 (SPSS $^{\circledR}$, Chicago, Illinois) was used for the statistical analyses.

\section{Results}

The SOL muscular activity between -60 and $0 \mathrm{~ms}$ in drop-jumps was significantly higher in condition CWNO than in condition CMO ( $p=.01$, Fig. 1$)$. In contrast, in the time window $120-180 \mathrm{~ms}$, the SOL EMG was lower in CWNO than in CMO $(p=.04)$. There was no significant difference in the muscular activation between CWNO and CMO in the time window $0-60 \mathrm{~ms}(p=.17)$ and also 60-120 ms $(p=.19)$, respectively (Fig. 1$)$.

Additionally, we investigated whether the overall muscular activation pattern of SOL changed dependent on the drop-jumping/landing condition (CWNO, CMO, $170 \mathrm{~ms}, 110 \mathrm{~ms}$ ). For drop-jumping, there was a significant effect for the interaction of the factors time window and condition, $F(9,72)=17.42, p<.001$ (factor time window: $F(3,24)=32.36, p<.001$; factor condition: $F(3,24)=95.15, p<.001$, Figs. 2 and 3, Table 1). For landing, the ANOVA revealed no significant effect 


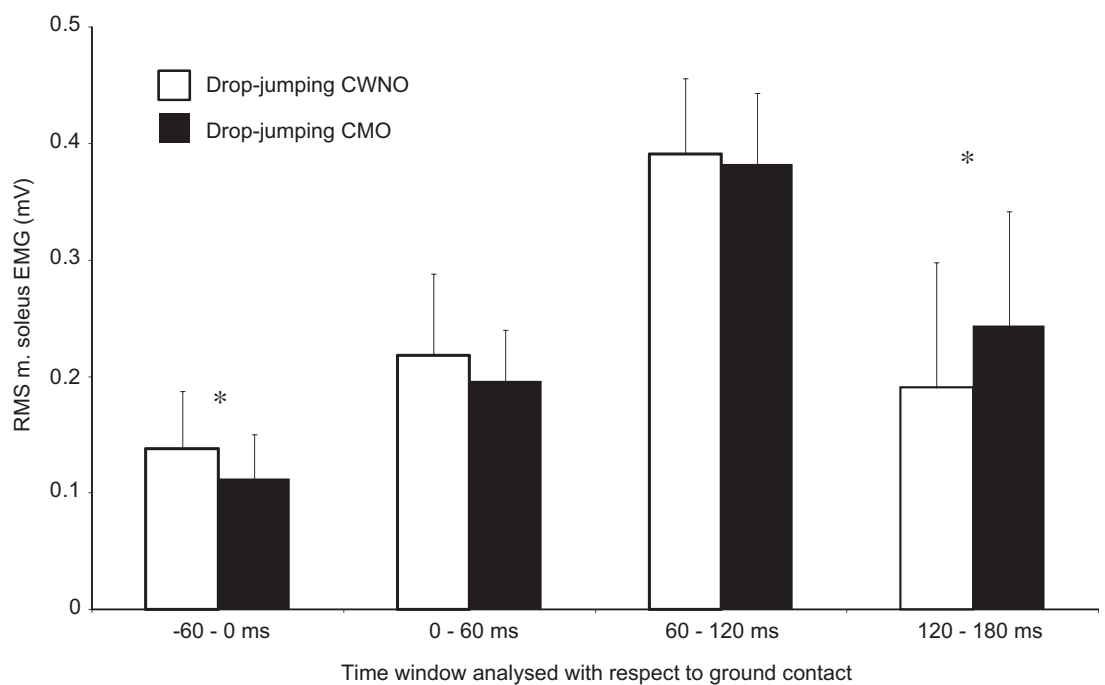

Fig. 1. Root mean square (RMS) values of the unrectified SOL EMG for the different time windows analyzed. Displayed are the condition (i) where subjects performed drop-jumps by knowing that there was no auditory cue (CWNO), and the condition (ii) where subjects performed drop-jumps where an auditory cue could be but was not presented (CMO). Statistical differences between the conditions are marked with asterisks. Black bars indicate the standard deviation (SD).

for the interaction of the factors time window and condition $(F(9,72)=1.65, p=.12$, factor time window: $F(3,24)=7.47, p<.01$; factor condition: $F(3,24)=2.98, p=.05$, Fig. 2$)$.

For the drop-jumps, it is possible that the modulated muscular activity in the different jump conditions might have changed the jump performance. Previous studies (Gollhofer \& Kyrolainen, 1991; Gollhofer, Strojnik, Rapp, \& Schweizer 1992) used the ground contact time (touch-down to push off) and the drop-jumping height as measures of performance. In the present study, the ANOVA revealed a trend towards a difference for the ground contact time $(F(3,27)=2.92, p=.05)$ but no differences in the drop-jumping height $(F(3,27)=0.96, p=.42)$. The mean values of the ground contact time of the different conditions were as follows: CWNO, $232 \pm 29 \mathrm{~ms}$; CMO, $252 \pm 42 \mathrm{~ms} ; 170 \mathrm{~ms}$, $253 \pm 42 \mathrm{~ms}$; $110 \mathrm{~ms}, 271 \pm 49 \mathrm{~ms}$. Accordingly, the mean values for the jump height were: CWNO, $24 \pm 3 \mathrm{~cm}$; CMO, $26 \pm 3 \mathrm{~cm} ; 170 \mathrm{~ms}, 25 \pm 4 \mathrm{~cm} ; 110 \mathrm{~ms}: 25 \pm 3 \mathrm{~cm}$.

\subsection{Ability to switch the movement}

With the intention to jump, subjects were unable to switch to landings in $14 \pm 16 \%$ of the trials if the auditory signal was presented $170 \mathrm{~ms}$ before ground contact, and in $39 \pm 24 \%$ of the trials at

Table 1

RMS grand mean values $(\mathrm{mV})$ ( \pm standard deviation, $S D$ ) of the SOL EMG for the different drop-jump and landing conditions.

\begin{tabular}{|c|c|c|c|c|c|c|c|c|c|}
\hline & & \multicolumn{4}{|c|}{ Drop-jump } & \multicolumn{4}{|l|}{ Landing } \\
\hline & & CWNO & CMO & $170 \mathrm{~ms}$ & $110 \mathrm{~ms}$ & CWNO & $\mathrm{CMO}$ & $170 \mathrm{~ms}$ & $110 \mathrm{~ms}$ \\
\hline \multirow[t]{2}{*}{$-60-0 \mathrm{~ms}$} & Mean & 0.14 & 0.11 & 0.11 & 0.10 & 0.11 & 0.11 & 0.12 & 0.11 \\
\hline & S.D. & 0.05 & 0.04 & 0.05 & 0.05 & 0.04 & 0.05 & 0.05 & 0.04 \\
\hline \multirow[t]{2}{*}{$0-60 \mathrm{~ms}$} & Mean & 0.22 & 0.20 & 0.19 & 0.15 & 0.09 & 0.12 & 0.13 & 0.14 \\
\hline & S.D. & 0.07 & 0.04 & 0.07 & 0.05 & 0.02 & 0.05 & 0.05 & 0.04 \\
\hline \multirow[t]{2}{*}{$60-120 \mathrm{~ms}$} & Mean & 0.39 & 0.38 & 0.37 & 0.35 & 0.15 & 0.17 & 0.16 & 0.20 \\
\hline & S.D. & 0.07 & 0.06 & 0.09 & 0.10 & 0.03 & 0.03 & 0.05 & 0.11 \\
\hline \multirow[t]{2}{*}{$120-180 \mathrm{~ms}$} & Mean & 0.19 & 0.24 & 0.26 & 0.32 & 0.08 & 0.08 & 0.08 & 0.13 \\
\hline & S.D. & 0.11 & 0.10 & 0.09 & 0.07 & 0.02 & 0.03 & 0.03 & 0.10 \\
\hline
\end{tabular}



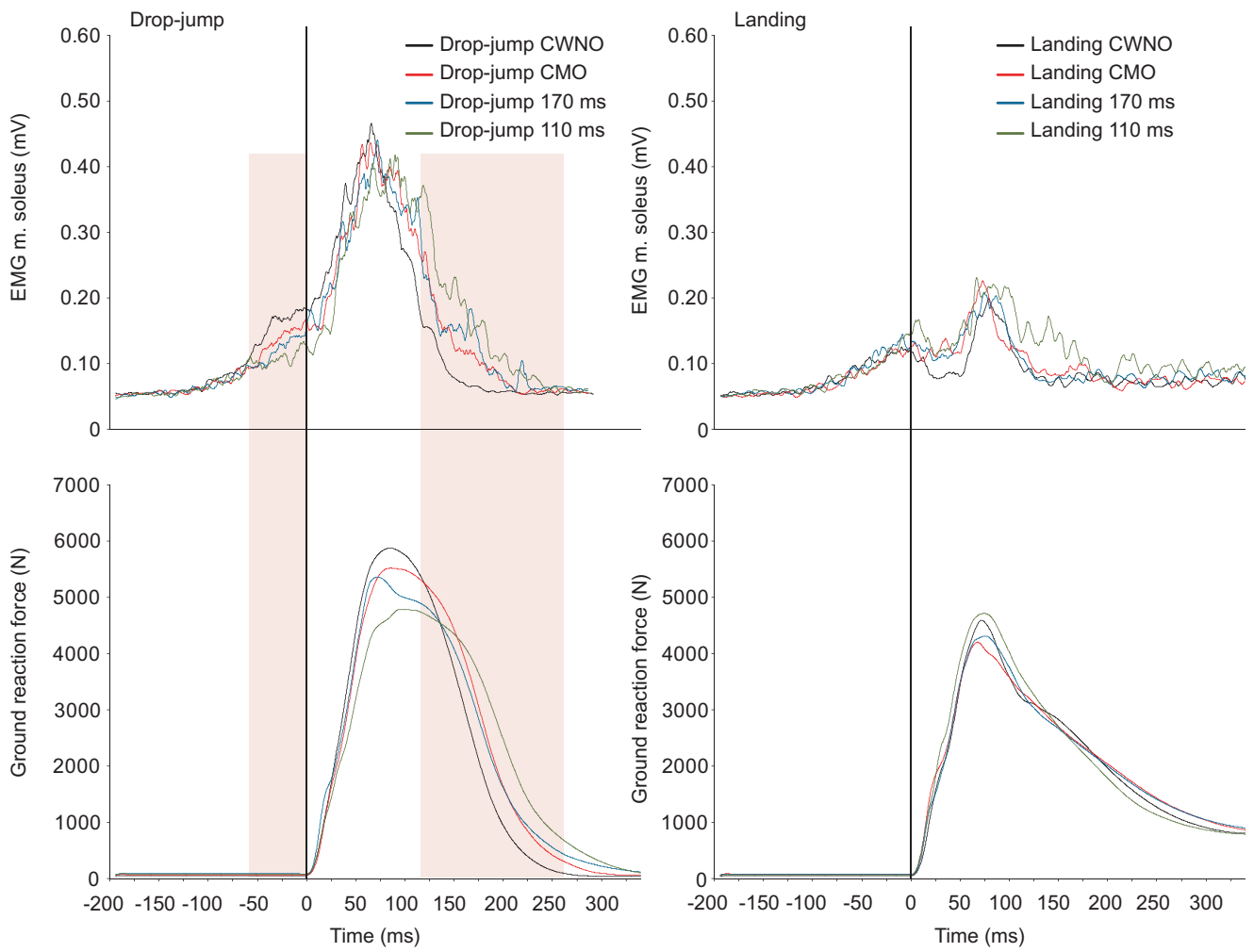

Fig. 2. Shown are grand mean values of the m. soleus EMG and the ground reaction force for all tested conditions. There were 4 conditions for both tasks, drop-jumps and landings: the first condition (termed "cue will not occur - CWNO") consisted of dropjumps/landings where the subjects knew that there will be no auditory cue. In the second condition, called "cue may occur CMO", the subjects performed the instructed movement (no switch of the movement) but they were aware that an auditory cue could be presented. The third and fourth condition, termed "170 ms" and "110 ms", respectively, refers to the landings/dropjumps where the movement was successfully switched based on the auditory cue presented at 170 and 110 ms before ground contact. Zero on the $x$-axis refers to ground contact. Data truncated at $340 \mathrm{~ms}$ following ground contact. The red boxes indicate phases of the drop-jumps where the muscular activation was clearly modulated depending on the performed condition.

110 ms. With the intention to land, subjects were unable to switch to drop-jumping in $14 \pm 8 \%$ at $170 \mathrm{~ms}$, and $31 \pm 28 \%$ at $110 \mathrm{~ms}$. As expected, in the $50 \mathrm{~ms}$ condition, the subjects were unable to switch the movement.

\section{Discussion}

\subsection{Changes in predictive motor control with uncertainties in task execution}

The main finding of the present study was an increased muscular activation just prior to touchdown in drop-jumps in the condition where subjects knew that they would not have to switch the task (CWNO) compared to the condition where the possibility existed that they had to switch to landing (CMO). In both conditions, subjects were principally instructed to perform drop-jumps and, in CMO, to switch to a landing movement with an auditory cue. The modulated muscular activity between CWNO and CMO prior to touch-down could only have occurred by changing predictive control. Thereby, it has to be noted that predictive control is often associated with an internal model of the central nervous system which controls the motor output and predicts the (sensory) consequences of the 


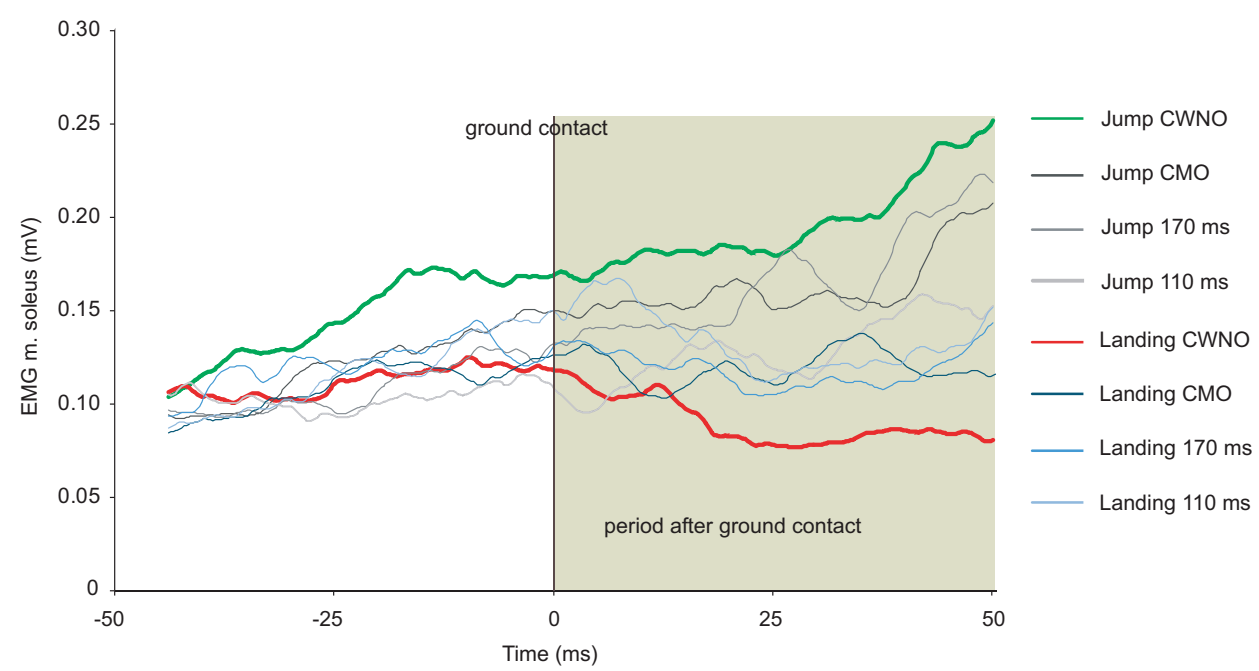

Fig. 3. Shown are the grand mean values of all tested drop-jump/landing conditions analyzed in the present study for a time window of $100 \mathrm{~ms}$ around the instant of ground contact. Note that, in all conditions where an auditory cue could be or was presented, the muscular activation was somewhere in the continuum between drop-jumping and landing with no cues (e.g., where the subjects knew that no auditory cue will be presented).

action(s) (Miall, Weir, Wolpert, \& Stein, 1993; Wolpert \& Miall, 1996). Consequently, to change prediction would mean to modify the internal model.

It is argued that the muscular activity was predictively modulated to adjust tendomuscular stiffness. In landing, kinetic energy has to be absorbed. In contrast, in drop-jumping, the ambition is to preserve as much kinetic energy as possible for the subsequent push-off. The absorption/preservation of energy can be achieved by adjusting the activity and therefore the stiffness of the tendomuscular system. Absorption of energy requires lower tendomuscular stiffness and thus lower muscular activity than the preservation (Dyhre-Poulsen et al. 1991; Hobara et al. 2007; Leukel et al., 2008). A further argument refers to time limitation. An immediate transfer from the eccentric to the concentric muscular action is a prerequisite in drop-jumps. This leaves less time for the eccentric phase than in landings. From a physical point of view, comparable kinetic energies in a shorter time produce higher forces which have to be counterbalanced by higher muscular activation/higher muscular stiffness. Transferred to the results of the present study this means that as soon as subjects were uncertain about whether to jump or to land, it was an adequate strategy for the central nervous system to lower the muscular activation prior to touch-down to meet the demands of the task that could have to be executed (e.g., to land). Importantly, and this is in contrast to all other conditions tested in the present study, there were actually no differences in sensory information (e.g., by the auditory cue, visual information) between conditions CWNO and CMO. Consequently, all changes of the muscular activation are based on adjustments in predictive motor control (changes of the internal model). Adjustments of this prediction may also have caused central changes of the processing of sensory feedback during the movement and, thus, modulation in reactive motor control after touch-down. For instants, the size of the H-reflex shortly after touch-down was shown to be altered with the falling height in drop-jumps (Leukel et al., 2008). This was hypothesized to reflect central (spinal) modulation of Ia afferent input (the Ia afferents being activated by muscle stretch at touch-down) by supraspinal centers finally causing adjustments of the reflex component in the EMG relative to the falling height. An adjustment of Ia afferent input to the motor neurons by supraspinal centers could have occurred in the present study.

With respect to the phases of the drop-jump after touch-down, it is interesting that the later concentric phase before jump-off was again different between CWNO and CMO. The muscular activation was significantly higher between 120 and $180 \mathrm{~ms}$ in the condition CMO than in the condition CWNO. It may be argued that the reduction in muscular activity and therefore tendomuscular stiffness in CMO 
was counterbalanced by an increased and prolonged (see Fig. 2) muscular activation during push-off. Pursuing the strategy of a intensified push-off prolonged the ground contract time in CMO but left the jumping height unchanged between the conditions (see also Hunter \& Marshall, 2002).

\subsection{Changes in muscular activation with the presence of an auditory cue}

Besides CWNO and CMO, there were other conditions tested in the present study where the subjects successfully switched from landing to drop-jumping upon auditory cues elicited prior to touch-down. An important finding of the analysis of the muscular activity of the 4 tested drop-jump conditions (CWNO, CMO, $170 \mathrm{~ms}, 110 \mathrm{~ms}$ ) was the graded motor output in the pre-activation phase prior to ground contact (see Fig. 2). The highest muscular activation in the eccentric phase occurred in the CWNO condition and the lowest in the $110 \mathrm{~ms}$ condition. The reason for the different muscular activities between CWNO and CMO was discussed above, e.g., subjects in the CMO condition in fact planned for a drop-jump but the probability to switch to landing caused them to decrease the muscular output. The reason for the even lower muscular activity in the $170 \mathrm{~ms}$ and $110 \mathrm{~ms}$ condition might be the time to integrate the sensory (auditory) information into the planning of the movement. In these 2 conditions, the subjects were instructed to plan for a landing and switch to a drop-jump with an auditory cue. Therefore, as soon as the auditory information was received, the motor command was changed. Drop-jumping requires higher muscular activity in the pre-activation phase than landing. This explains why the muscular activity was lower in the $110 \mathrm{~ms}$ condition than in the $170 \mathrm{~ms}$ condition, because the cue was presented closer to touch-down. Finally, when the cue was presented $50 \mathrm{~ms}$ before ground contact, time constraints prevented that the motor command could be changed.

As a consequence of the lower muscular activation prior to ground contact, additional muscular activity in the late concentric phase may have compensated for the decreased muscular stiffness, meaning that SOL was more and longer active in the $110 \mathrm{~ms}$ condition than in the CWNO condition.

\subsection{Differences in the modulation of muscular activity between drop-jumps and landings}

The modulations of the muscular activity with respect to the presence and the timing of the auditory cue were observable for drop-jumps but not landings. One reason for the differences between landings and drop-jumps might be the complexity of the movement control. Specifically, it might be less difficult to prepare for a landing motor pattern than to prepare for a drop-jump.

Another reason could be that other (leg) muscles not tested in the present study changed their pattern with the different delays of the auditory cues.

\subsection{Functional considerations}

There is a growing interest to design training interventions with the focus that people develop motor skills to prevent injuries. Drop-jump training is one example where one important aim is to improve motor control in order to reduce the incidence of injuries during sports games (for review, see Renstrom et al., 2008). Most of these training interventions consist of protocols where isolated tasks have to be trained. In daily life, injuries occur very seldom while performing an isolated task in a predictable environment but rather in uncertain environments. The results of the present study showed that the muscular pattern is modified as soon as there are uncertainties with respect to task execution compared to when the subjects are certain about what task they have to execute. Consequently, when developing training interventions to prevent injuries in daily life, it might be beneficial to consider and integrate the aspect of uncertainty.

\section{Acknowledgments}

We are grateful to the Landesgraduiertenförderung who supported part of this work in terms of a scholarship to Christian Leukel. In addition, this work was supported by the Bundesinstitut für Sportwissenschaft (BiSp, Germany) and the Bundesamt für Sport (BASPO, Switzerland). 


\section{References}

Bastian, A. J. (2006). Learning to predict the future: The cerebellum adapts feedforward movement control. Current Opinion in Neurobiology, 16, 645-649.

Dyhre-Poulsen, P., Simonsen, E. B., \& Voigt, M. (1991). Dynamic control of muscle stiffness and H-reflex modulation during hopping and jumping in man. Journal of Physiology, 437, 287-304.

Gollhofer, A., \& Kyrolainen, H. (1991). Neuromuscular control of the human leg extensor muscles in jump exercises under various stretch-load conditions. International Journal of Sports Medicine, 12, 34-40.

Gollhofer, A., Strojnik, V., Rapp, W., \& Schweizer, L. (1992). Behaviour of triceps surae muscle-tendon complex in different jump conditions. European Journal of Applied Physiology and Occupational Physiology, 64, 283-291.

Hobara, H., Kanosue, K., \& Suzuki, S. (2007). Changes in muscle activity with increase in leg stiffness during hopping. Neuroscience Letters, 418, 55-59.

Hunter, J. P., \& Marshall, R. N. (2002). Effects of power and flexibility training on vertical jump technique. Medicine and Science in Sports and Exercise, 34, 478-486.

Jones, G. M., \& Watt, D. G. (1971). Observations on the control of stepping and hopping movements in man. Journal of Physiology, 219, 709-727.

Komi, P. V. (1984). Biomechanics and neuromuscular performance. Medicine and Science in Sports and Exercise, 16, 26-28.

Komi, P. V. (2003). Stretch-shortening cycle. In P. V. Komi (Ed.), Strength and power in sport (pp. 184-202). Oxford: Blackwell Science.

Komi, P. V., \& Gollhofer, A. (1997). Stretch reflex can have an important role in force enhancement during SSC-exercise. Journal of Applied Biomechanics, 13, 451-460.

Leukel, C., Gollhofer, A., \& Taube, W. (2008). Phase- and task-specific modulation of soleus H-reflexes during drop-jumps and landings. Experimental Brain Research, 190, 71-79.

Leukel, C., Lundbye-Jensen, J., Gruber, M., Zuur, B., Gollhofer, A., \& Taube, W. (2009). Short-term pressure induced suppression of the short latency response - A new methodology for investigating stretch reflexes. Journal of Applied Physiology, 107 1051-1058.

Leukel, C., Taube, W., Gruber, M., Hodapp, M., \& Gollhofer, A. (2008). Influence of falling height on the excitability of the soleus H-reflex during drop-jumps. Acta Physiologica (Oxf.), 192, 569-576.

Miall, R. C., Weir, D. J., Wolpert, D. M., \& Stein, J. F. (1993). Is the cerebellum a Smith predictor? Journal of Motor Behavior, 25, 203-216.

Petersen, N., Christensen, L. O., Morita, H., Sinkjaer, T., \& Nielsen, J. (1998). Evidence that a transcortical pathway contributes to stretch reflexes in the tibialis anterior muscle in man. Journal of Physiology, 512, 267-276.

Renstrom, P., Ljungqvist, A., Arendt, E., Beynnon, B., Fukubayashi, T., Garrett, W., et al. (2008). Non-contact ACL injuries in female athletes: An international olympic committee current concepts statement. British Journal of Sports Medicine, 42, $394-412$.

Sherrington, C. S. (1910). Flexion-reflex of the limb, crossed extension-reflex, and reflex stepping and standing. Journal of Physiology, 40, 28-121.

Wolpert, D. M., \& Miall, R. C. (1996). Forward models for physiological motor control. Neural Networks, 9, 1265-1279.

Zuur, A. T., Lundbye-Jensen, J., Leukel, C., Taube, W., Gollhofer, A., Nielsen, J. B., et al. (2010). Contribution of afferent feedback and descending drive to human hopping. Journal of Physiology, 588, 799-807. 